\title{
Current insights into protease dynamics in human epithelial disease and barrier function
}

\author{
Michael A. Curtis • David P. Kelsell
}

Published online: 17 January 2013

(C) Springer-Verlag Berlin Heidelberg 2013

The fundamental importance of proteolytic enzymes to the biology of all living organisms is manifestly demonstrated by genomic analyses which demonstrate that genes encoding proteases and their inhibitors account for over $2 \%$ of the coding sequences of most genomes sequenced to date. For example, over 500 protease genes have been annotated in the human genome. This extraordinary diversity reflects the wide range of biological functions performed by this family of enzymes: in addition to relatively non-specific degradative functions, characterized for example by digestive enzymes of the intestine, proteases also perform the selective and limited cleavage of a myriad of protein substrates which defines their subsequent biological functions and properties. In so doing, protease-dependent peptide bond hydrolysis ranks among the most significant of all posttranslational modification systems and underpins the processes of development, tissue homeostasis, turnover and remodelling, immune protection and healing. Given their central role in health, it is unsurprising that imbalances in protease activity are also intimately associated with both inherited and acquired human conditions.

Proteases are generally classified on the basis of their mechanism of peptide bond cleavage. Four major families are well known. In the case of serine and cysteine enzymes, the hydroxyl and thiol residues respectively act as the nucleophilic groups in catalyzing hydrolytic peptide bond cleavage. Metallo- enzymes, utilize a metal ion, frequently $\mathrm{Zn}^{2+}$, at their active site to activate a molecule of water to act as the nucleophile attack group. In aspartyl, sometimes

\section{A. Curtis • D. P. Kelsell $(\square)$}

Blizard Institute, Barts and the London School of Medicine and Dentistry, Queen Mary University of London, Whitechapel, London E1 2AT, UK

e-mail: d.p.kelsell@qmul.ac.uk

M. A. Curtis

e-mail:m.a.curtis@qmul.ac.uk referred to as acidic, proteases a similar mechanism of action to the metallo-enzymes is proposed in which a molecule of water is co-ordinated in the active site between two conserved aspartic acid residues, one of which causes activation of the water by removal of a proton and formation of the nucleophilic group required for peptide bond cleavage. In addition to these well known major catalytic classes, a number of additional mechanisms of peptide bond cleavage are now recognized leading to an expanded classification system which includes three additional classes.

A subset of acidic proteases was originally identified based on their resistance to pepstatin, a broad spectrum inhibitor of proteases active at acidic $\mathrm{pH}$. The unusual active sites of this pepstatin-insensitive subset proved difficult to characterise, but it is now recognized that some members of the subset were actually misclassified serine proteases, but with an acidic $\mathrm{pH}$ optimum, whilst the remainder represented a new class of protease containing glutamic acid/glutamine in their active site rather than aspartic acid. A further catalytic type of protease was identified following the elucidation of the crystallographic structure of the yeast proteasome which demonstrated the presence of an $\mathrm{N}$-terminal threonine at the reactive centre fulfilling a similar nucleophilic role to the serine residue in the active sites of serine proteases. Once again this helped explain an inhibitor resistance phenomenon in that the proteasome was well known to be insensitive to inhibition by a wide range of standard protease inhibitors. The final and most recent addition to the list of classes is unusual in that members of this group are not proteases at all: they do not achieve peptide bond through hydrolysis, but instead act through the specific and unusual chemistry of asparagine. An asparagine residue at the active site is able to act as a nucleophile and cause autolytic cleavage of the peptide chain. In this instance the asparagine residue is destroyed and therefore the active site functions only once. The reader is referred to the MEROPS database (http://merops.sanger.ac.uk) which provides an excellent on 
line resource of hierarchical protease classification based on these basic categories of catalytic mechanism using amino acid sequence and structure comparisons (Rawlings et al. 2012).

Peptide bond hydrolysis by proteases is a defining feature of the biology of all organisms. However, it is becoming increasingly apparent from the analysis of genomes that the expression of pseudo-proteases, products of protease encoding genes that have evolved to generate an inactive form, is a very common phenomenon. The mechanism through which these inactive proteases exert their biological effect is an area of significant current research activity. One potential property may relate to regulatory functions because of the ability of these "dead" enzymes to bind to their cognate substrate without mediating proteolysis in addition to the contribution of non-peptidase domains on the molecule to their overall biological function. In so doing they may provide a protective function, mediate interaction with other ligands or play a role in intra- or extra-cellular localization.

This volume of reviews highlights the role of proteases and protease inhibitor systems in the maintenance of health and development of disease at the epithelial barriers of the skin and mucosal surfaces and draws upon examples of several of the catalytic types of enzymes and inactive forms described above. The first series of articles highlights the biological importance of proteolysis to the normal processes of development, homeostasis and repair. Rawlings and Voegli describe the role of proteases in the stratum corneum and the regulatory mechanisms that are essential for the maintenance of the normal properties of skin (Rawlings and Voegli 2013). In particular the pivotal role of the LEKTI family of inhibitors and the central role they perform in the control of many of the proteases of the stratum corneum is described. This paper also neatly emphasizes the critical issue of the requirement for balance between proteases and their cognate inhibitors. Delayed desquamation leading to accumulation of corneocytes on the surface of the SC can be attributed to a reduced level of proteolytic processing of the corneodesmosome. In this instance an imbalance in favour of lowered proteolysis in the stratum corneum leads to the condition of dry skin. Conversely an increased overall level of proteolysis is present in inflammatory conditions of the skin typified by psoriasis and barrier abnormalities resulting from premature desquamation. Brocklehurst and Philpott also emphasize the critical role played by proteolytic enzymes in desquamation and in particular focuses upon the involvement of two cysteine proteases, cathepsin V and cathepsin L (Brocklehurst and Philpott 2013). The authors describe how inhibition of these enzymes by cystatin is responsible for the regulation of processing of transglutaminases and thereby control of cross-linking and desquamation of the stratum corneum. The importance of proteases to the development of epithelial tissues is further explored in the paper by Miller and List who describe how the co-ordinated action of a protease cascade is responsible for the development of the barrier function of the stratum corneum (Miller and List 2013). In this instance, the cascade is initiated by the epidermal serine protease, matriptase, which activates a membraneanchored second, serine protease, prostasin. Activated prostasin then orchestrates the down-stream effectors of the cascade to ensure appropriate barrier development. Loss of either protease in mice presents the same phenotype of impaired corneocyte differentiation and lipid matrix formation together with loss of tight junction development and consequent neonatal lethality. The role of the cascade is not limited to in utero development, however, since loss of function of matriptase in adult mice influences the function of a range of epithelial types in different tissues. Understanding the regulation of this proteolysis system is therefore pertinent to both development and to normal function in adults.

The role of proteolytic enzymes in wound healing is addressed (Martins et al. 2013). In particular, the authors focus upon the variety of functions performed by members of the matrix-metallo protease (MMP's) family. In addition to remodeling of the extracellular matrix, these enzymes are now recognized to have a broader significance to the overall co-ordination of the repair process through their actions on growth factor activation, cell recruitment and cell-cell or cell-matrix signaling. In so doing MMP's, and their cognate inhibitors are at the very centre of the highly co-ordinated process required to restore tissue integrity following injury. The theme of extracellular matrix remodeling is also described (Biancheri et al. 2013), this time in the context of the mucosal barrier of the intestine. Once again the contribution of proteolysis to the regulation of tissue remodeling is emphasized by the potentiation of the activity of signaling molecules and their receptors and through effects on elements of the innate immune response including antimicrobial peptides. As described previously in the case of the stratum corneum, deregulation of these normally homeostatic proteolytic actions is a hallmark of immune/inflammatory disorders of the intestine. In this instance proteasemediated disruption at this boundary leads to further exacerbation of the inflammatory process because of the increased antigen access to the underlying tissues which drives further damaging immune responses.

In the next series of papers, the influence of loss of specific gene function in diseases characterised by skin barrier dysfunction and deregulated protease activity is described. Scott et al review the biological role of ATPbinding cassette transporter A12 (ABCA12) in skin-barrier permeability (Scott et al. 2013). The importance of this lipid transporter to lamellar membrane formation and the development of the skin barrier is dramatically evidenced by the devastating effects of loss of function of ABCA12 seen in the autosomal recessive skin disease, Harlequin Ichthyosis. 
The authors also discuss how the abnormal lamellar granule formation in this disease may cause aberrant production of granule associated proteases involved in the desquamation process and associated effects on the skin barrier. In Netherton syndrome (Hovnanian 2013), loss of function mutations in a serine protease inhibitor, lympho-epithelial Kazal type related inhibitor type 5 (LEKTI-1) leads to uncontrolled activity of a series of serine proteases involved in skin barrier formation, specifically kallikrein-related peptidase (KLK) 5, KLK7, KLK12 and elastase 2. The net result of the unopposed activity of these enzymes is stratum corneum detachment, due to cleavage of desmogelin-2, activation of the pro-inflammatory responses through cleavage of protease activated receptor (PAR) 2 and processing of prointerleukin- $1 \beta$ to the active cytokine. Finally, the elucidation of the functions of the rhomboid proteases and their inactive homologues is described. As discussed previously proteases which have lost their proteolytic activity during evolution retain key non-protease dependent functions, frequently involved in regulation. Inactive rhomboid proteases, iRhoms, are a good example and Etheridge et al describe the likely role of iRhom-2 in the regulation of epidermal growth factor signaling- which is itself a substrate of RHBDL2, an active rhomboid protease- either directly or via ADAM17 regulation (Etheridge et al. 2013). Dominant mutations in iRHOM2 underlie a hyperproliferative skin disease (termed tylosis) linked with a dramatic susceptibility to cancer of the oesophagous.

The final series of papers deals with the influence of exogenously-derived proteases on perturbation of the epithelial barrier. Proteolytic enzymes from bacteria and parasites on our skin and mucosae present a particularly difficult problem to the maintenance of homeostasis at these barriers as these enzymes are normally not recognized by our endogenous protease inhibitors and hence can act in an unopposed manner. Thibodeau and Butterworth describe the consequences of the activity of bacterial proteases in the cystic fibrotic lung, a site which is also characterized by widespread dysregulation of host derived proteolytic enzymes (Thibodeau and Butterworth 2013). The combined effect of this uncontrolled proteolysis is highlighted with particular reference to the activity of the epithelial sodium channel $(\mathrm{ENaC})$ which is a key molecule in cystic fibrosis disease. Koziel and Potempa summarise the roles of proteolytic enzymes produced by two bacterial pathogens of the skin, Staphylococcus aureus and Streptococcus pyogenes, in the breach of the skin barrier and evasion of the normally protective immune mechanisms at this boundary (Koziel and Potempa 2013). Specifically, secreted enzymes from both these organisms are capable of the degradation of proteins of the connective tissues of the dermis and activation of the inflammatory response through the contact system, kinin release and potentially via PAR activation on keratinocytes. The ensuing inflammatory reaction may be withstood by these bacteria through their ability to disarm key elements of the host defence, including neutrophils, chemokines, antimicrobial peptides and complement. In so doing, these organisms may develop an appropriate site in the skin for entry into the deeper tissues and subsequent spread of infection. Last, the paper by Holt et al describes the complex evolution of the digestive enzymes of free-living and parasitic mites (Holt et al. 2013). These intestinal enzymes have been shown to have both allergic and inflammatory properties on the mucous membranes of humans most notably in the case of the actions of the cysteine proteases derived from the house dust mite on the respiratory epithelium.

In summary, the epithelial barrier is, in part, regulated by a complex interaction between proteases and their inhibitors. They play key roles in response to physical injury, bacterial infection or disease. Major clues as their individual functions and regulation are being further elucidated from human genetic disease associations.

\section{References}

Biancheri P, Di Sabatino A, Corazza GR, MacDonald TT (2013) Proteases and the gut barrier. Cell Tissue Res. doi:10.1007/ s00441-012-1390-z

Brocklehurst K, Philpott MP (2013) Cysteine proteases: mode of action and role in epidermal differentiation. Cell Tissue Res. doi:10.1007/s00441-013-1557-2

Etheridge SL, Brooke MA, Kelsell DP, Blaydon DC (2013) Rhomboid proteins: a role in keratinocyte proliferation and cancer. Cell Tissue Res. doi:10.1007/s00441-012-1542-1

Holt DC, Burgess STG, Reynolds SL, Mahmood W, Fischer K (2013) Intestinal proteases of free-living and parasitic astigmatid mites. Cell Tissue Res. doi:10.1007/s00441-012-1369-9

Hovnanian A (2013) Netherton syndrome: skin inflammation and allergy by loss of protease inhibition. Cell Tissue Res. doi:10.1007/s00441-013-1558-1

Koziel J, Potempa J (2013) Protease-armed bacteria in the skin. Cell Tissue Res. doi:10.1007/s00441-012-1355-2

Martins VL, Caley M, O'Toole EA (2013) Matrix metalloproteinases and epidermal wound repair. Cell Tissue Res. doi:10.1007/ s00441-012-1410-z

Miller GS, List K (2013) The matriptase-prostasin proteolytic cascade in epithelial development and pathology. Cell Tissue Res. doi:10.1007/s00441-012-1348-1

Rawlings ND, Barrett AJ, Bateman A (2012) Nucleic Acids Res 40: D343-D350, MEROPS: the database of proteolytic enzymes, their substrates and inhibitors

Rawlings AV, Voegeli R (2013) Stratum corneum proteases and dry skin conditions. Cell Tissue Res. doi:10.1007/s00441-012-1501-x

Scott CA, Rajpopat S, Di W-L (2013) Harlequin ichthyosis: ABCA12 mutations underlie defective lipid transport, reduced protease regulation and skin-barrier dysfunction. Cell Tissue Res. doi:10.1007/s00441-012-1474-9

Thibodeau PH, Butterworth MB (2013) Proteases, cystic fibrosis and the epithelial sodium channel $(\mathrm{ENaC})$. Cell Tissue Res. doi:10.1007/s00441-012-1439-z 